\title{
Evolution of penetration mechanism induced by strain rate effect
}

\author{
Gan $\mathrm{Li}^{1,2, *}$, Chunming Song ${ }^{2}$, and Mingyang Wang ${ }^{1,2}$ \\ ${ }^{1}$ Nanjing University of Science and Technology, School of Mechanical Engineering, Nanjing, China; \\ ${ }^{2}$ Army Engineering University of PLA, State Key Lab. of Disaster Prevention \& Mitigation of Explosion \& Impact, Nanjing, China
}

\begin{abstract}
According to the dynamic mechanical properties, in the striking velocity range, the strain rate of the projectile and target caused by penetration can reach $10^{4} \sim 10^{6} / \mathrm{s}$. The strain rate effect increases sharply and then tends to saturation. During the penetration process, the mechanical properties of the target and the projectile change violently and present serious spatial inhomogeneity, which has a great influence on the penetration effect. In this paper, penetration experiments of granite targets by steel projectiles are carried out in the range of $1200 \mathrm{~m} / \mathrm{s} \sim 2400 \mathrm{~m} / \mathrm{s}$, the crater parameters are measured and the projectiles are recovered to obtain the macroscopic failure pattern of the projectiles and the targets. Based on the dynamic mechanical properties, an interaction model of the projectiles and the target is established, which considers the spatial and temporal distributions of the strain rates during penetration. With this model, the influences of material mechanical behaviour on penetration effect at different velocities are analysed, the formation cause and internal mechanism of penetration effect are discussed, and the influence mechanism of the strain rate effect on the penetration mechanism evolution is also revealed.
\end{abstract}

\section{Introduction}

With the rapid development of high velocity / hypervelocity kinetic energy weapons, the attack velocity of the projectiles is in the range of $300 \mathrm{~m} / \mathrm{s}$ to $3000 \mathrm{~m} / \mathrm{s}$ for different targets. The kinetic energy weapons have great damage to the protection works, which not only cause structural damage, but also produce strong impact effects, which is the key and difficult problem in national defense for all countries [1-3].

Based on the dynamic responses of the projectile and the target, the penetration effect can be divided into low velocity zone, high velocity zone and hyper-velocity zone, corresponding to different penetration mechanisms [4]. In this paper, we only pay attention to the targets of rock and concrete. In the low velocity zone, the projectile can be treated as rigid approximately, and the cavity expansion model which is developed based on this hypothesis can predict the penetration depth effectively $[5,6]$. In the hyper-velocity zone, the stress-strain relationship and the state equation of the projectile and target materials tend to be fluid, and penetration mechanism becomes fluid penetration. The AT model and its modified models $[7,8]$ are becoming more and more perfect in the description of this stage. In the high velocity zone, which is between the low velocity zone and hyper-velocity zone, the projectile is deformed with substantial mass loss, and the penetration mechanism is changed from rigid penetration into fluid penetration which is usually referred to as semi fluid penetration. In this range, for rock type targets, the nonlinear effect of constitutive equation and state equation are very obvious due to the large deformation and the fracture expansion.
The penetration phenomenon is more complex than the other two stages, and the penetration depth will even decrease by contraries [9-14]. In the references the interpretation of this phenomenon is that, the deformation of the projectile induces the diameter of the projectile increasing and the resistance increases as well, while the mass of the projectile is constant during the penetration process [16-19]. However, it is not consistent with the experimental results obtained in this paper, which needs further study.

According to the dynamic mechanical properties, in the striking velocity range, the strain rate of the projectile and target caused by penetration can reach $10^{4} \sim 10^{6} / \mathrm{s}$. The strain rate effect increases sharply and then tends to saturation. During the penetration process, the mechanical properties of the target and the projectile change violently and present serious spatial inhomogeneity, which has a great influence on the penetration effect. In this paper, penetration experiments of granite targets by steel projectiles are carried out in the range of $1200 \mathrm{~m} / \mathrm{s} \sim 2400 \mathrm{~m} / \mathrm{s}$, the crater parameters are measured and the projectiles are recovered to obtain the macroscopic failure pattern of the projectiles and the targets. Based on the dynamic mechanical properties, an interaction model of the projectiles and the target is established, which considers the spatial and temporal distributions of the strain rates during penetration. With this model, the influences of material mechanical behavior on penetration effect at different velocities are analyzed, the formation cause and internal mechanism of penetration effect are discussed, and the influence mechanism of the strain rate effect on the penetration mechanism evolution is also revealed.

Corresponding author: ligan1205@163.com 


\section{Penetration experiments}

\subsection{Experimental system}

The experiment is based on a 100/30 mm two light gas gun, which can accelerate a projectile of $90 \mathrm{~g}$ to $3 \mathrm{~km} / \mathrm{s}$. The experimental system is shown in Figure 1, the velocity of the launched projectile is measured using the laser beam interruption method (error less than 1\%). When the sabots of the projectile are separated, the projectile finally hit the granite target in the target chamber. The striking velocity is controlled by adjusting the pressure in the high pressure chamber and the pump tube. After the experiment, the macro destructions of the targets are observed to study the trajectory development and the penetration depth, and the projectiles are recovered to measure the deformation and quality.

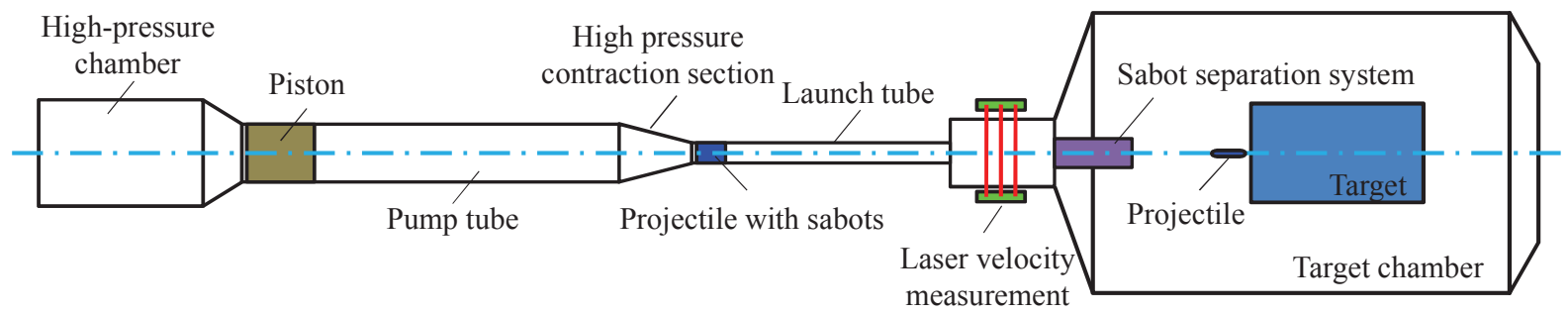

Fig. 1. Schematic of experimental system.

The ogive-nose projectile used in the experiment is shown in Figure 2, the full length, $L_{0}$, is $54 \mathrm{~mm}$; the diameter, $d_{0}$, is $10.8 \mathrm{~mm}$; the ratio of length to diameter, $L_{0} / d_{0}$, is 5 ; the caliber radius head, $\mathrm{CRH}$, is 3.0 ; and the initial mass of the projectile, $m_{p}$, is $32.45 \mathrm{~g}$. The projectile is made of high strength alloy steel $30 \mathrm{CrMnSiNi2A}$ with a hardness of HRC50 after heat treatment, and its basic material properties are shown in Table 1.

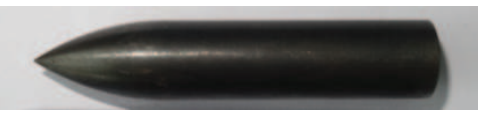

Fig. 2. The projectile used in the experiment.

Table 1. Projectile parameters.

\begin{tabular}{|c|c|c|c|c|}
\hline Material & $\begin{array}{c}\text { Density } \\
/ \mathbf{g} / \mathbf{c m}^{3}\end{array}$ & $\begin{array}{c}\text { Quality } \\
\mathbf{g}\end{array}$ & $\begin{array}{c}\text { Uniaxial compressiv } \\
\text { strength / MPa }\end{array}$ & Hardness \\
\hline 30CrMnSiNi2A & 7.85 & 32.45 & 1952 & HRC50 \\
\hline
\end{tabular}

The target is the granite produced in Wulian County, Shandong. The target body is constrained by a cylindrical steel sleeve, with a diameter of $480 \mathrm{~mm}$ and a wall thickness of $10 \mathrm{~mm}$. The granite is a rectangular block of $300 \mathrm{~mm} \times 300 \mathrm{~mm} \times 800 \mathrm{~mm}$, and the gap between them is filled with $\mathrm{C} 40$ concrete. The basic material properties of the granite are shown in Table 2.

Table 2. Main performance parameters of granite target.

\begin{tabular}{|c|c|c|c|c|}
\hline Material & $\begin{array}{c}\text { Density } \\
\text { g/cm3 }\end{array}$ & $\begin{array}{c}\text { Uniaxial static } \\
\text { compressive } \\
\text { strength /MPa }\end{array}$ & $\begin{array}{c}\text { Dynamic } \\
\text { compression } \\
\text { strength / MPa }\end{array}$ & $\begin{array}{c}\text { Elastic } \\
\text { modulus } \\
\text { / GPa }\end{array}$ \\
\hline $\begin{array}{l}\text { Wulian } \\
\text { granite }\end{array}$ & 2.67 & 147 & $240^{*}$ & 54.6 \\
\hline \multicolumn{2}{|c|}{ the parameter was obtained by SHPB experiments in the strain rate of $37 \sim 63 \mathrm{~s}^{-}$}
\end{tabular}

the parameter was obtained by SHPB experiments in the strain rate of $37 \sim 63 \mathrm{~s}^{-}$

\subsection{Experimental results}

Penetration experiments were carried out 12 times, the velocities were in the range of $1.2 \mathrm{~km} / \mathrm{s}$ to $2.4 \mathrm{~km} / \mathrm{s}$ and the results were shown in table 3 .

Table 3. Experimental results.

\begin{tabular}{|c|c|c|c|}
\hline $\begin{array}{c}\text { Exp. } \\
\text { Number }\end{array}$ & $\begin{array}{c}\text { Striking } \\
\text { velocity / m/s }\end{array}$ & $\begin{array}{c}\text { Penetration } \\
\text { depth / mm }\end{array}$ & $\begin{array}{c}\text { Qualityof the } \\
\text { recovered } \\
\text { projectiles /g }\end{array}$ \\
\hline 1 & 1196 & 118.80 & 31.65 \\
\hline 2 & 1426 & 146.02 & 31.42 \\
\hline 3 & 1430 & 155.80 & 31.32 \\
\hline 4 & 1600 & 163.90 & 30.83 \\
\hline 5 & 1654 & 134.00 & 30.83 \\
\hline 6 & 1752 & 87.40 & 10.17 \\
\hline 7 & 1789 & 83.10 & 9.35 \\
\hline 8 & 1808 & 93.40 & 10.25 \\
\hline 9 & 2067 & 105.47 & 6.36 \\
\hline 10 & 2165 & 104.73 & 5.19 \\
\hline 11 & 2356 & 109.84 & 5.13 \\
\hline 12 & 2378 & 101.48 & 3.79 \\
\hline
\end{tabular}

Li and Song [20] have described the macroscopic damage form of the projectile and the target in detail. In this paper, the depth and mass loss of the projectile are analyzed only. The relationship between the projectile velocity and the ratio of the penetration depth to the projectile length is shown in Figure 3, and the corresponding mass loss rates are also drawn in the figure.

In the velocity range from 1200 to $1640 \mathrm{~m} / \mathrm{s}$, the penetration depth increases lineally, and the mass loss rate of the projectile is less than $5 \%$ which is linear with the initial impact kinetic energy. The projectile-head 
recovered is not obviously blunted compared with the initial projectile. Therefore, it can be considered that the penetration mechanism is rigid penetration. In the range from 1640 to $1800 \mathrm{~m} / \mathrm{s}$, the penetration depth decreases sharply, and the mass loss rate increases sharply to $70 \%$; the penetration phenomenon in the stage is significantly different with the rigid penetration which belongs to the semi-fluid penetration. When the velocity is between 1800 and $2400 \mathrm{~m} / \mathrm{s}$, the penetration depth increases slowly again, but shows obvious nonlinearity. At this stage, the mass of the recovered projectile decreases further, and the projectile is almost abraded completely.

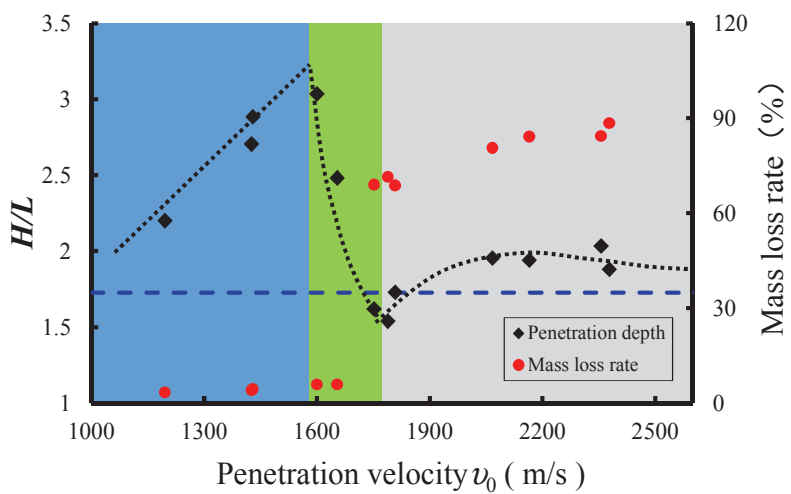

Fig.3. The penetration depth and the mass loss rate of the projectile.

As a reference, the hydrodynamic limit of the penetration depth is shown in Figure 3 with a dotted line. According to the AT model, for the strength of the projectile used in this paper is much higher than that of the target, we can predict a new penetration peak at the stage of fluid penetration. Limited by the experimental conditions, our results have shown the upward trend only.

\section{Distribution of strain rate}

\subsection{Dynamic expanding cavity model}

The response zones formed in the target during a penetration can be divided into elastic, cracked and comminuted and undisturbed zones, as shown in Fig. 4. The cavity expansion velocity is determined by the normal component of the projectile velocity. The boundary of the comminuted zone is determined by the uniaxial compressive strength of the target, and the boundary of the cracked zone is determined by the tensile strength of the target, the stress at the boundary of the elastic zone is zero.

According to conservations of mass and momentum:

$$
\left\{\begin{array}{l}
\frac{\partial \rho}{\partial t}+\frac{1}{r^{2}} \frac{\partial}{\partial t}\left(\rho r^{2} v\right)=0 \\
\frac{\partial \sigma_{r}}{\partial r}+\frac{2\left(\sigma_{r}-\sigma_{\theta}\right)}{r}=-\rho\left(\frac{\partial v}{\partial t}+v \frac{\partial v}{\partial r}\right)
\end{array}\right.
$$

where $\sigma_{r}$ and $\sigma_{\theta}$ are radial stress and circumferential stress respectively, $v$ is radial particle velocity. If the incompressible assumption is adopted, formula (1) can be simplified to:

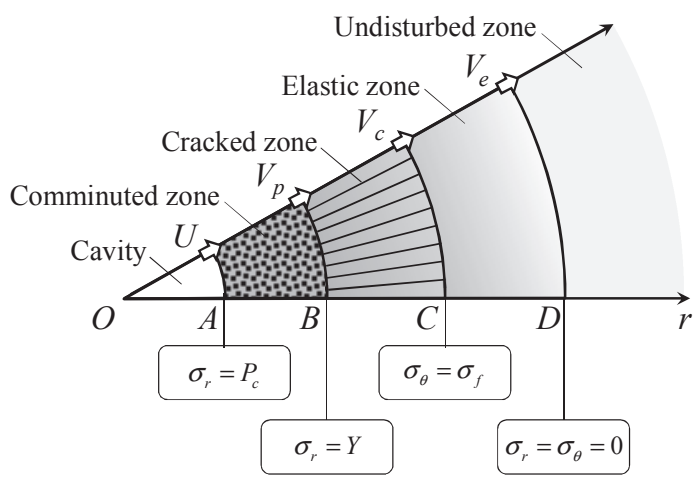

Fig.4. Schematic of the dynamic expanding cavity model.

$$
\frac{\partial v}{\partial r}+\frac{2 v}{r}=0
$$

In dynamic expanding cavity model, the response of the comminuted zone influences the penetration effect most significant. According to formula (3), the particle velocity satisfies the following relationship:

$$
\frac{1}{r^{2}} \frac{\partial}{\partial t}\left(\rho r^{2} v\right)=0
$$

Using the velocity boundary conditions of the cavity expansion surface, the particle velocity distribution is:

$$
v=\frac{U(U t)^{2}}{r^{2}}
$$

where $U$ is the cavity expansion velocity.

\subsection{Strain rate distribution of the target}

The relationship between particle velocity, $v$, and displacement, $u$, is as following:

$$
v=\frac{\mathrm{d} u}{\mathrm{~d} t}=\frac{\partial u}{\partial t}+v \frac{\partial u}{\partial r}
$$

According to the definition of true strain and strain rate, we can get:

$$
\dot{\varepsilon}_{r}=-\frac{\partial v}{\partial r}, \quad \dot{\varepsilon}_{\theta}=-\frac{v}{r}
$$

Using formula (7), the strain rate in the comminuted zone is as following:

$$
\dot{\varepsilon}_{r}=\frac{2 U(U t)^{2}}{r^{3}}, \quad \dot{\varepsilon}_{\theta}=-\frac{U(U t)^{2}}{r^{3}}
$$

It can be seen that the strain rate has a significant relationship with the geometrical size of the projectile. The distribution of the strain rate induced by different warhead sizes is shown in Figure 5. At the same penetration speed, the smaller the radius of curvature of the projectile is, the larger the strain rate is, and the smaller the influence range is. For the projectile with a radius of $10 \mathrm{~mm}$, the maximum strain rate is about $10^{6}$ $/ \mathrm{s}$, and the strain rate decreases to $10^{3} / \mathrm{s}$ at the distance from the impact point $50 \mathrm{~mm}$. 


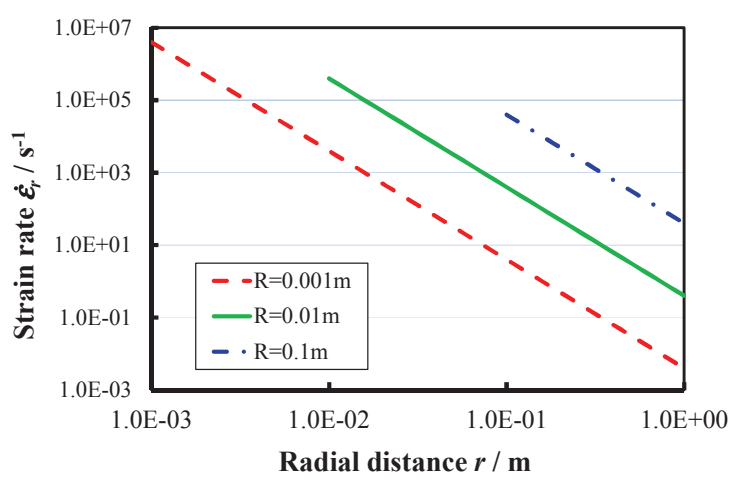

Fig.5. Strain rate distribution of the target when $v=2000 \mathrm{~m} / \mathrm{s}$.

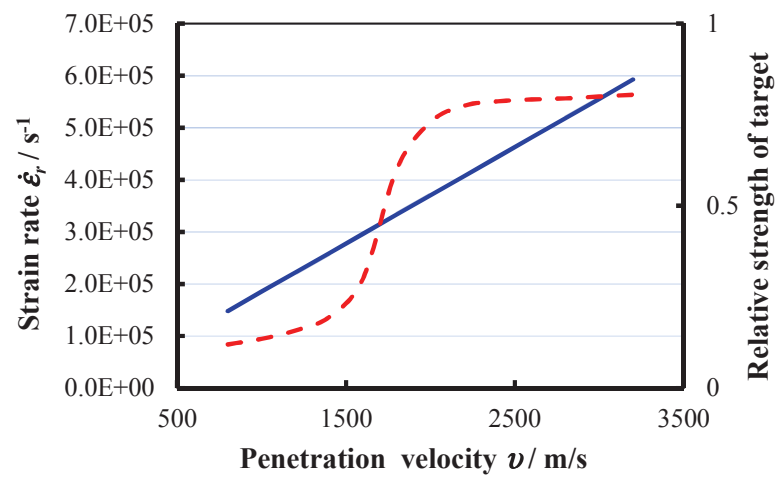

Fig.6. Strain rate and relative strength of target under different striking velocity.

The strain rate of the target under different striking velocity is shown in Figure 6, when the diameter of the projectile is $10.8 \mathrm{~mm}$. The strain rate increases linearly with the velocity, and the order of magnitude is $10^{5} / \mathrm{s}$ in the range from 1000 to $3000 \mathrm{~m} / \mathrm{s}$. For the target material, $10^{5} / \mathrm{s}$ is the critical window in which the strain rate effect changes sharply and tends to be saturated. Figure 6 shows the relative strength of the target under ultra-high strain rate qualitatively. It can be seen that within a very narrow velocity range, the resistance of the projectile will increase rapidly, resulting in reducing of the penetration depth contrarily.

\section{Conclusion and discussion}

In this paper, we have carried out the penetration experiments with granite targets and ogive-nose projectiles at striking velocities between 1.2 and 2.4 $\mathrm{km} / \mathrm{s}$. By analyzing the factors such as the penetration depth of the target, the mass loss rate of the projectile and the strain rate distribution induced by the penetration, the following conclusions are drawn:

(1) As the striking velocity increasing, the penetration depth shows the three trend of the first linear increase, then the sharp decline and then the nonlinear growth, which corresponds to rigid penetration, semi-fluid penetration and fluid penetration.

(2) There is obvious size effect in the strain rate distribution caused by penetration, and the attenuation radius of the order of magnitude is 2.2 times that of the radius of the projectile.
(3) At the stage of semi-fluid penetration, the resistance of the target to the projectile increases sharply, resulting in the reverse of the penetration depth.

Further work will focus on the properties of the projectile at ultra-high strain rate and the internal friction effect of the powder in the comminuted zone, to reveal the mechanism of the interaction of projectile/target.

\section{References}

1. L J Zhang, D S Liu, C G Yu, et al. Aero weaponry, 2, 13-16 (2010)

2. F Harry. Int. J. of Impact Engineering, 5: 1-4 (1987)

3. Q M Zhang, F L Huang. Introduction of Hyper velocity impact dynamics. (Beijing: Science press: 2000)

4. Y H Cheng, M Y Wang, C C Shi, et al. J. Zhejiang Univ. 49(4), 616-625 (2015).

5. Forrestal M J, Altman B S, Cargile J D, et al. Int. J. Impact Eng, 15(4), 395-405 (1994)

6. D Z Yankelevsky. Int. J. Impact Eng. 106, 30-43 (2017)

7. A Tate. J. Mech. Phys. Solids, 15(6): 387-399 (1967)

8. G F Gao. Sci. \& tech. review, 34(2): 287-298 (2016)

9. M J Forrestal, D J Frew, S J Hanchak, et al. Int. J. Impact Eng. 18, 465-76 (1996)

10. D J Frew, M J Forrestal, S J Hanchak. ASME J. Appl. Mech. 67, 841-5 (2000)

11. A F Savvateev, A V Budin, V A Kolikov, et al. Int. J. Impact Eng. 26: 675-681 (2001)

12. M J Forrestal, A J Piekutowski. Int. J. Impact Eng. 24, 57-67 (2000)

13. A J Piekutowski, M J Forrestal, K L Poormon, et al. Int. J. Impact Eng. 23(1): 723-734 (1999)

14. M Wickert. Proc 23rd Int Symp Ballistics, (Tarragona, Spain, 2007)

15. D Z Zhang, X R Zhang, J D Lin, et al. Chinese J. Rock Mech. Eng. 24, 1612-1618 (2005)

16. X W Chen, Q M Li. ASCE J. Eng. Mech. 130(1), 123-127 (2004)

17. S B Segletes. Int. J. Solids Struct, 44(7e8), 21682191 (2007)

18. H M Wen, B Lan. Acta Mech. Sin. 26, 573-583 (2010)

19. Z Rosenberg, E Dekel. Int. J. Solids and Struct. 47, 238-250 (2010)

20. G Li, C M Song, Y Y Qiu, et al. Chinese J. Rock Mech. Eng. 37(1): 61-66 (2018) 